Meta

Journal des tradlucteurs

Translators' Journal

\title{
Traduction biblique et genèse linguistique
}

\section{Henri Van Hoof et Samia Barrada}

Volume 35, numéro 4, décembre 1990

URI : https://id.erudit.org/iderudit/003093ar

DOI : https://doi.org/10.7202/003093ar

Aller au sommaire du numéro

Éditeur(s)

Les Presses de l'Université de Montréal

ISSN

0026-0452 (imprimé)

1492-1421 (numérique)

Découvrir la revue

Citer cette note

Van Hoof, H. \& Barrada, S. (1990). Traduction biblique et genèse linguistique. Meta, 35(4), 795-798. https://doi.org/10.7202/003093ar d'utilisation que vous pouvez consulter en ligne.

https://apropos.erudit.org/fr/usagers/politique-dutilisation/ 


\section{BLOC-NOTES}

\section{TRADUCTION BIBLIQUE ET}

GENĖSE LINGUISTIQUE

«Les traducteurs sont les chevaux de trait de la civilisation.» Appliquée à la traduction biblique, cettc pensée de Joseph de Maistre prend sa pleine signification. La traduction biblique, en effet, possède la tradition la plus longue et la plus continue de tous les types de traduction en Occident. Elle débute au III 
siècle avant notre ère avec la traduction de l'Ancien Testament de l'hébreu en grec pour la communaute judéo-grecque d'Égypte. Sur ordre du roi Ptolémée II Philadelphe (308-246 av. J.-C.), soixante-douze Juifs «de noble réputation», qui maîtrisaient les langues hébraïque et grecque, furent envoyés par le Grand Prêtre de Jérusalem en Égypte pour s'atteler ensemble à la traduction que l'on connâit sous le nom de Septuaginte. Elle servit au II ${ }^{\mathrm{e}}$ siècle de notre ère à la première version latine, la Vetus Latina, qui incorporait déjà le Nouveau Testament, dont tous les livres avaient été rédigés en grec. C'est cette version que saint Jérôme fut chargé de réviser en 382 , avant de l'abandonner pour se lancer dans une traduction entièrement sienne, la Vulgate, réalisée entre 392 et 410.

Vers la mêrne époque déjà, d'autres traducteurs se mettaient à l'cuvre pour propager la Parole parmi les peuples d'Occident, dont certains ne possédaient encore ni langue écrite, ni alphabet. D'emblée, la traduction biblique allait devoir se doubler d'un travail linguistique considérable, auquel certaines cultures sont redevables, les unes de la naissance de leur langue écrite, les autres du développement de leur langue littéraire.

Dans la seconde moitié du IVe siècle, un prêtre arien du nom de Wulfila (311-383), né de père wisigoth et de mère cappadocienne, fuyant les persécutions du roi wisigoth Athanaric, installe sa communauté dans la province romaine de Mésie, entre le Danube et les Balkans (l'actuelle Bulgarie). Fait évêque par Eusèbe de Nicomédie, il devient l'apôtre des Goths et voit dans la traduction des Écritures l'instrument indispensable de leur christianisation. Mais le gotique, rameau oriental des idiomes germaniques, n'est alors que langue parlee et la première difficulté à laquelle se heurte Wulfila est donc celle d'inventer un alphabet pour en transcrire les sons. De culture grecque et latine, il forme cet alphabet à l'aide de caractères empruntés au grec et au latin et complétés par des idéogrammes runiques pour la transposition des sons gotiques inconnus de la langue grecque $^{1}$. Cet outil lui permet d'entreprendre enfin la traduction projetée, pour laquelle il se base sur un texte grec qu'il rend mot à mot, en suivant l'ordre des mots et la syntaxe du grec, souvent obligé de créer des mots composés dont chaque élément est traduit séparément du grec.

Par cette traduction, terminée vers 370 , Wulfila se pose en fondateur d'une littérature gotique grâce à laquelle on peut suivre l'évolution de la langue germanique durant quinze siècles. De la langue conçue par lui, des termes sont passés dans tous les idiomes germaniques, tel le verbe fastan, «tenir bon» en gotique, auquel Wulfila donne le sens religieux de «jeûner» et que l'on retrouve avec cette signification en néerlandais (vasten), en anglais (to fast), en allemand (fasten), etc.

Un peu plus tard, au début du Ve siècle, et un peu plus au sud, en Arménie, une aventure assez semblable survient à l'ancien conseiller royal Mesrop
Machtots (350-441), devenu moine (382) puis évêque (390). Depuis le VIe siècle avant notre ère, l'Arménie vivait dans l'orbite du monde perse et c'est pour lutter contre le mazdéisme intolérant des rois sassanides que les chefs spirituels du pays fraîchement christianisé (250-330) décident de traduire la Bible dans la langue du peuple. Mais, là aussi, le premier obstacle vient de l'absence de langue livresque. Mesrop Machtots, homme de grande science, se charge de créer un alphabet (405) et, en collaboration avec le patriarche Sahag Partev, donne la première version arménienne de la Bible (405-409) pour laquelle ils sont partis d'un texte en syriaque, qui était langue de culte au même titre que le grec. Cette traduction se caractérise par la pureté d'une langue riche et expressive et par la distinction du style. L'invention de l'alphabet et la traduction biblique ont entraîné un tel essor des lettres que le Ve siècle a mérité d'être appelé l'âge d'or de la littérature arménienne.

Quand, au IX ${ }^{\mathbf{e}}$ siècle, de l'autre côté de la Mer Noire, l'évêque Cyrille et son frère Méthode, moine dans un monastère de l'Olympe, répondant à l'injonction de l'empereur byzantin Michel III, quittent leur Macédoine natale pour aller convertir les peuplades slaves établies de la Dalmatie à la Pologne, ils ne se doutent certainement pas que leur action va déclencher un processus dont l'aboutissement ne sera rien de moins que l'enfantement de la langue russe. Cyrille (827-869) et Méthode (828-885), originaires de la région de Salonique, avaient pour langue matemelle le slavon ou vieux-slave (ou vieuxbulgare), qui reposait sur un parler de la Macédoine méridionale. C'est le plus ancien connu des dialectes slaves, mais jusqu'à l'époque des deux évangélisateurs il n'était pas écrit. Or, pour s'embarquer dans leur mission, Cyrille et Méthode se veulent armés de la Parole divine et décident de traduire le Nouveau Testament, le Psautier, le Sacramentaire et quelques autres textes sacrés du grec en slavon. Leur première tâche consiste à trouver un système permettant de noter les sons de la langue parlée. C'est ainsi qu'ils mettent au point l'alphabet glagolitique (de glagol parole, en slavon), basé sur la minuscule grecque stylisée, et complété par quelques signes d'origine orientale. Réformé par des disciples inconnus des apôtres de la Moravie, il est remplacé par l'alphabet cyrillique, qui est tiré de la capitale grecque et bientôt le seul à subsister, celui dont va se servir la langue russe ultérieurement. Car si, grâce à la traduction de Cyrille et Méthode, le slavon a acquis ses lettres de noblesse dès le $\mathrm{IX}^{\mathrm{e}}$ siècle, les premières traductions en vieux-russe ne feront leur apparition que deux siècles plus tard. C'est la fusion du slave ecclésiastique et de la langue populaire qui, vers le $\mathrm{XI}^{\mathrm{e}}$ siècle, donne naissance au vieux-russe, lequel à son tour engendre, dans la période du XII ${ }^{e}$ au XIVe siècle, le biélorusse, l'ukrainien ou petit-russe et le russe proprement dit ou grand-russe. Cyrille et Méthode auraient été heureux de voir leur travail de pionniers récompensé par le fait que le premier livre imprimé en russe fut les Actes des Apôtres (1554). 
Les traducteurs bibliques n'ont pas tous été dans l'obligation de concevoir d'abord un alphabet avant de pouvoir accomplir leur cuvre. Pour certains, qui disposaient déjà d'une langue écrite, le mérite est de l'avoir élevée d'un idiome informe au rang de véritable langue de culture. Tel est le cas, notamment, de l'anglais et de l'allemand.

En Grande-Bretagne, jusqu'à la fin du XIV siècle, l'anglais demeure une langue inférieure aux yeux des lettrés et des savants. Ce n'est qu'en 1399, date à laquelle le roi Henry II l'emploie pour la première fois au Parlement, qu'elle supplante officiellement le latin. Le théologien John Wyclif (13241384), après avoir écrit en latin jusqu'en 1380 , se met alors à l'anglais pour traduire la Bible, se réservant le Nouveau Testament et, peut-être, une partic de l'Ancien, celui-ci étant surtout l'œuvre de Nicholas Hereford, son coadjuteur, et de John Purvey. Il existe deux versions de la Bible de Wyclif, toutes deux basées sur la Vulgate: la première guindée et craignant de s'écarter du latin, la seconde plus libre et osant être anglaise. Malgré ses imperfections, cette première traduction intégrale des Ecritures en vulgaire n'en a pas moins jeté les fondements de la langue biblique anglaise et contribué grandement au développement de la prose.

L'humanisme remet le latin en honneur, mais la traduction vient au secours de la langue anglaise par le biais des controverses de la Réforme qui, très tôt, influencent la vie intellectuelle. Le réformateur gallois William Tyndale (1494-1536) forme le projet d'une nouvelle traduction de la Bible pour ses coreligionnaires et entreprend le Nouveau Testament à Londres, en 1523. Mais, à une époque où la royauté est encore attachée à Rome, il craint pour sa tranquillité et s'embarque pour Hambourg, visite Luther à Wittenberg et commence l'impression de sa traduction à Cologne. Contraint de se réfugier à Worms, il y achève et imprime la version intégrale du Nouveau Testament en 1525. Ensuite, Tyndale se rend à Marbourg où il donne, en 1530, le Pentateuque et, en 1531, le Livre de Jonas. En 1533, l'hostilité de Henry VIII s'étant apparemment atténuée, il se hasarde à mettre le cap sur Anvers, mais, alors qu'il travaillait à la révision de ses traductions, il est trahi et livré à la police impériale en 1535 . Conduit au château de Vilvorde, il y est pendu et brûlé l'année suivante. Ses traductions sont celles d'un érudit sensible à l'harmonie des mots. Ses connaissances lui permettant de ne pas dépendre uniquement de la Vulgate, il s'est inspiré aussi de la version allemande de Luther et des versions grecque et latine commentées par Êrasme. Au moment de sa mort, il avait déjà bien entamé l'Ancien Testament et fixé à jamais le caractère de la langue biblique anglaise.

Son œuvre fut achevée en 1535 par le moine augustin Miles Coverdale (1488-1568) et publiée sous le titre Biblia, the Bible: that is the Holy Scripture of the Olde and New Testament faithfully and newly translated out of the Doutche ${ }^{2}$ and Latyn into English pour être adoptée - ô ironie du sort - par Henry
VIII lui-même qui avait entre-temps rompu avec la papauté. En 1537, Thomas Matthew donne une nouvelle version qui combine Tyndale et Coverdale. Celle de Taverner, en 1539, n'en est qu'une révision, de même d'ailleurs que la Great Bible (1539-41). En 1567, le Nouveau Testament est retraduit par William Whittingham, tandis qu'une nouvelle version de la Bible entière, due à la collaboration de Whittingham avec John Knox, Anthony Gilby et Thomas Sampson, est dédiée à Elizabeth I. En 1568 paraît encore la Bishop's Bible, réalisée par un collège d'évêques à l'initiative de l'archevêque de Canterbury, Matthew Parker. Par le truchement de ces multiples traductions, les textes sacrés pénètrent dans toutes les maisons et forment contrepoids aux œuvres gréco-latines de l'humanisme. Ecrites dans une langue simple, sans pédanteries mais aussi sans trivialité, elles fusionnent parfaitement les éléments saxons et français, créant une prose biblique dont l'influence sera prépondérante sur toutes la littérature anglaise.

En Allemagne comme en Angleterre, l'humanisme se teinte très vite de connotations religieuses, car dès 1519 le réformateur Martin Luther (14831546) proclame la seule autorité du jugement individuel appliqué à l'étude de la Bible. Le mouvement a aussi des conséquences linguistiques, car s'il puise son inspiration dans l'Antiquité et se prononce en faveur du latin, la Réforme au contraire prend résolument parti pour la langue du peuple. À l'époque, il n'existe encore qu'une série de dialectes très différenciés; seules les chancelleries font usage d'un allemand passe-partout dont l'administration des princes et des villes assure l'adaptation aux dialectes locaux. Et pourtant, estime Luther, le peuple doit pouvoir lire les Écritures dans sa langue. Certes, on possédait des versions partielles en haut-allemand, en bas-allemand et en alémanique du siècle précédent, mais il rêvait d'une traduction intelligible pour tous. Ce qu'il réussit à faire en partant de la langue et du style des chancelleries saxonnes. La traduction du Nouveau Testament, commencée en décembre 1521, ne lui prend que trois mois et paraît à Wittenberg en 1522 sous le titre Das Neue Testament Teutsch. Pour celle de l'Ancien Testament, beaucoup plus laborieuse bien que Luther se soit mis à l'étude du grec, des amis lettrés, parmi lesquels Melanchthon (Philipp Schwarzerd, 1497-1560), lui viennent en aide, de sorte que la Bible complète - Biblia, das ist die gantze heilige Schrifft Deudsch - peut voir le jour en 1534. Le fait même d'incorporer le mot deutsch au titre de sa traduction le fait passer d'un concept jusque-là abstrait à une réalité tangible pour le peuple tout entier.

La qualité de la traduction luthérienne en a fait un monument d'une importance primordiale pour la littérature allemande dans son ensemble. Le principal, en effet, n'est pas qu'il ait donné de la Bible une interprétation nouvelle, mais bien comment il l'a fait et dans quelle langue. En donnant corps à l'atmosphère biblique, en suscitant les conditions spirituelles nécessaires à l'éclosion d'une poésie nouvelle, en prouvant la maniabilité de la langue vulgaire dans les 
sujets les plus élevés, Luther a véritablement jeté les bases de l'allemand littéraire. Par sa volonté de créer une langue commune à tous les Allemands, il a fait cuvre de linguiste, normalisant la transcription phonétique, fixant l'orthographe, redressant les formes grammaticales, réalisant ainsi, en moins d'un demisiècle, l'unification d'une langue écrite qui se propagea rapidement: entre 1518 et 1525 parurent plus de livres en allemand que dans les cinquante années qui avaient suivi la découverte de l'imprimerie.

Note

1. L'alphabet de Wulfila ne doit pas être confondu avec l'écriture gothique allemande, qui n'est qu'une transformation de l'alphabet latin.

2. Doutche $=$ Deutsch $=$ allemand

HENRI VAN HOOF Bruxelles, Belgique 